\title{
Laboratory Diagnosis of Childhood Tuberculosis: is Stool an Appropriate Sample?
}

\author{
Ashok Rattan* \\ Ex Professor and Head, Department of Microbiology and Molecular Biology, Mahatma Gandhi Medical College and Advisor: Laboratory \\ Operations Pathkind Diagnostics, Gurugram, India \\ *Corresponding Author: Ashok Rattan, Ex Professor and Head, Department of Microbiology and Molecular Biology, Mahatma Gandhi \\ Medical College and Advisor: Laboratory Operations Pathkind Diagnostics, Gurugram, India.
}

Received: September 03, 2019; Published: September 16, 2019

DOI: 10.31080/ASMI.2019.02.0376

The World Health Organization (WHO) estimates that over half a million new cases of childhood tuberculosis (TB) occur every year, with an annual mortality of up to 80,000 ., most of the deaths occurring in children below 5 years of age and before initiation of treatment. Childhood TB is guesstimated to account for $10-15 \%$ of the TB cases worldwide and up to $25 \%$ of cases from high TB burden countries. TB can progress very rapidly in children because of their immature immune system. High suspension and Rapid detection of TB in children should enable more rapid treatment to be initiated leading to improved outcomes [1].

Because of the myriad ways in which TB can present, TB disease should be suspected in any child with epidemiologic risk factors and suggestive symptoms or signs of organ involvement that are not explained by other diagnoses. Clinical suspicion is critical. Diagnosis is aided by diagnostic imaging, ancillary laboratory tests and by microbiologic confirmation. Positive results for tuberculin skin tests and interferon gamma release assays may support the diagnosis of TB disease, but results may be negative in as many as $30 \%$ of children with TB disease according to a meta-analysis pediatric TB is often a paucibacillary disease: results for acid-fast bacillus stains are frequently negative and, despite best efforts, cultures may be negative for over $40 \%$ of children with TB disease. Diagnosis in children is often made on the basis of known contact with an infectious source case, a positive tuberculin skin test or interferon gamma release assay and characteristic abnormalities in chest radiography. Children with a high pretest probability of TB disease require treatment even if the cultures are negative; this especially applies to suspected TB meningitis, because serious complications, such as stroke, may develop rapidly [2].

$\mathrm{TB}$ in children is mostly intrathoracic (pulmonary) in $>75 \%$ of cases, and is mainly paucibacillary (smear-negative). Current bacteriologic tests to confirm TB in children have limited sensitivity and resource-intensive methods are needed to collect adequate samples. Current available tests confirm a minority of children with TB: $5-10 \%$ of children with TB are smear microscopy posi- tive and $10-40 \%$ culture positive depending on disease severity and spectrum, resulting in a perceived unfavorable cost-benefit ratio for bacteriologic testing in most resource-limited settings. The Xpert MTB/RIFC assay (Xpert; Cepheid, Sunnydale, CA), endorsed by the World Health Organization for the diagnosis of TB and rifampicin resistance in adults and children including in resourcelimited settings in 2013, yields rapid results, but has $<70 \%$ sensitivity compared to culture in children. Diagnosis of tuberculosis in children, therefore, is a major challenge. Infants and young children may cough, but they cannot be instructed to expectorate the sputum that they produce. If they cough, they usually swallow the sputum. Xpert also requires appropriate samples in young children, such as gastric aspirates (GA), induced sputum (IS), nasopharyngeal aspirates (NPA), fine needle aspiration (FNA) of peripheral lymph nodes or bronchoalveolar lavage (BAL), all resource-intense, invasive procedures $[3,4]$.

In developing regions, an increasing proportion of these children are co-infected with HIV, which results in more severe TB disease and higher mortality. Children often acquire their disease from infected adults in their own family or in the community. In fact, the incidence of TB in children, who usually present with symptoms within a year of infection, provides a snapshot of transmission within a community [5].

Diagnostic and management challenges in children are due to the paucibacillary nature of tuberculosis disease, challenges of obtaining respiratory samples, and the wide spectrum of disease manifestations and severity that often overlap with other common childhood conditions such as pneumonia, HIV associated lung disease, and malnutrition. Depending on the study setting and resources, microbiological confirmation is established by culture in only $15 \%-50 \%$ of pediatric cases. Although the recently endorsed Xpert MTB/RIF assay is more sensitive and specific than smear microscopy in children with tuberculosis, it only has a sensitivity of approximately $66 \%$ on respiratory specimens compared with culture. For these reasons, the diagnosis of intrathoracic tuberculosis 
in children frequently is based on nonspecific symptoms and signs, supported by evidence of exposure to a tuberculosis case or infection with Mycobacterium tuberculosis, and on chest radiography. The limitations of this diagnostic approach are well recognized. It is universally acknowledged that improving the diagnosis of tuberculosis in children both through the development of novel diagnostics and by optimizing the use of current tools in necessary to decrease mortality and morbidity from TB in children [6].

In 2013, WHO endorsed the use of Xpert as the initial diagnostic test in all children suspected of having TB, both for expectorated sputum and samples obtained via gastric lavage. But in children only $15 \%$ with TB have smear-positive respiratory samples. Their organisms tend to stay in the perihilar lymph nodes and rarely rupture into the bronchial tree, as they do in adults. Consequently, children's respiratory samples may have few or no mycobacteria to detect regardless of the system used. Children older than 8 years old are more likely to exhibit adult-like pulmonary TB with coughing and culture-positive sputum. Thus today, diagnosis of active tuberculosis in children, particularly those $<8$ years old, is based on a combination of clinical, radiological, and laboratory criteria.

In high burden low-income and middle-income countries (LMIC), TB in children remains underdiagnosed with only $45 \%$ of the estimated number of cases notified to the WHO in 2016. In many of these countries, rising levels of drug resistance makes it essential to achieve the highest possible rates of microbiological confirmation in order to tailor the treatment. For microbiology based diagnosis critical to select the best specimen collection method, especially in children who have difficulties in producing sputum spontaneously. The most advanced method, the bronchoalveolar lavage, is not available in many LMIC. Other methods, such as induced sputum (IS), gastric aspirate (GA) or nasopharyngeal aspirate (NPA) are potential good alternatives [3].

In a study in UK, it was reported that use of 3 induced sputum samples was more sensitive than use of 3 gastric washings for diagnosis of tuberculosis in patients who could not expectorate spontaneously. Use of bronchoscopy with bronchoalveolar lavage did not increase diagnostic sensitivity. Samples could be collected in 1 day, allowing for faster diagnosis, faster initiation of treatment, and shorter hospital stay [7].

\section{Sputum induction}

IS specimens need to be obtained in negative- pressure isolation rooms. Staff protection measures included use of appropriate respiratory protection masks, limitation of the time spent in the room during the procedure, and use of standard hospital procedures for staff exposed to TB. For each IS procedure, $30 \mathrm{~mL}$ of
$3 \%$ hypertonic saline was administered via a mouthpiece using an ultrasonic nebulizer (Devilbiss Ultraneb 99 or Respiflo Aerodyne Omega Plus). Typically, $1-20 \mathrm{~mL}$ of sputum was obtained over the subsequent $1-2 \mathrm{~h}$ (the volume was recorded by the nurse performing IS),

Both IS and GA are recommended by WHO for diagnosis of ITB in children unable to produce sputum. However, in most LMIC, these methods are poorly implemented, especially at secondary or primary healthcare settings due to operational challenges. IS requires adequate training of health workers, equipment and hygiene precautions and GA requires admission of the child. Improving diagnostic capacity at secondary and primary healthcare centres is crucial to increase case detection. NPA might be an easier and better accepted method compared with IS or GA, but its implementation would require optimization of equipment (aspirator) and material (mucus extractor). Recently, a great interest emerged from the use of stool to detect TB bacilli that are swallowed for the diagnosis of childhood TB. Despite variable sensitivity of Xpert on stools $(32 \%-85 \%)$ between studies, and laboratory challenges to find the best sample processing method to remove the PCR inhibiting factors common in stool, this specimen could be a good alternative to sputum in children. Authors have shown that Xpert on the combination of stool and NPA could achieve similar detection yields as 2SI or 2GA, offering a good opportunity for decentralization of the microbiological diagnosis of childhood TB. The use of Xpert Ultra cartridge that has a level of detection close to the one of culture with the upcoming GeneXpert Omni Point of Care platform combined to more child friendly specimens collection methods like NPA and stools could significantly improve the microbiological diagnostic capacity of childhood ITB in LMIC but the logistic implications in programmatic conditions will need to be assessed [3].

For comparison against the microbiological reference standard, sensitivities of stool Xpert varied from $32 \%$ to $85 \%$, while specificity was uniformly very high. (Forest PlotA). The pooled sensitivity was $67 \%$ and pooled specificity was $99 \%$. For the clinical reference standard comparison, the pooled sensitivity of stool Xpert was $22 \%$ while the specificity was $100 \%$ (Forest PlotB).

The recently developed next-generation Xpert MTB/RIF Ultra (GX-Ultra; Cepheid) assay aims to overcome the limitations of its predecessor by increasing the sensitivity for detection of MTB DNA when few bacilli are present in a clinical sample. This new and fully automated nested real-time PCR assay differs from its predecessor in several ways: the larger PCR chamber with a total capacity of $50 \mathrm{ul}$, in contrast to $25 \mathrm{ul}$ in the previous cartridge, the incorporation of two different multicopy targets (IS1081 and IS6110 inser- 
A
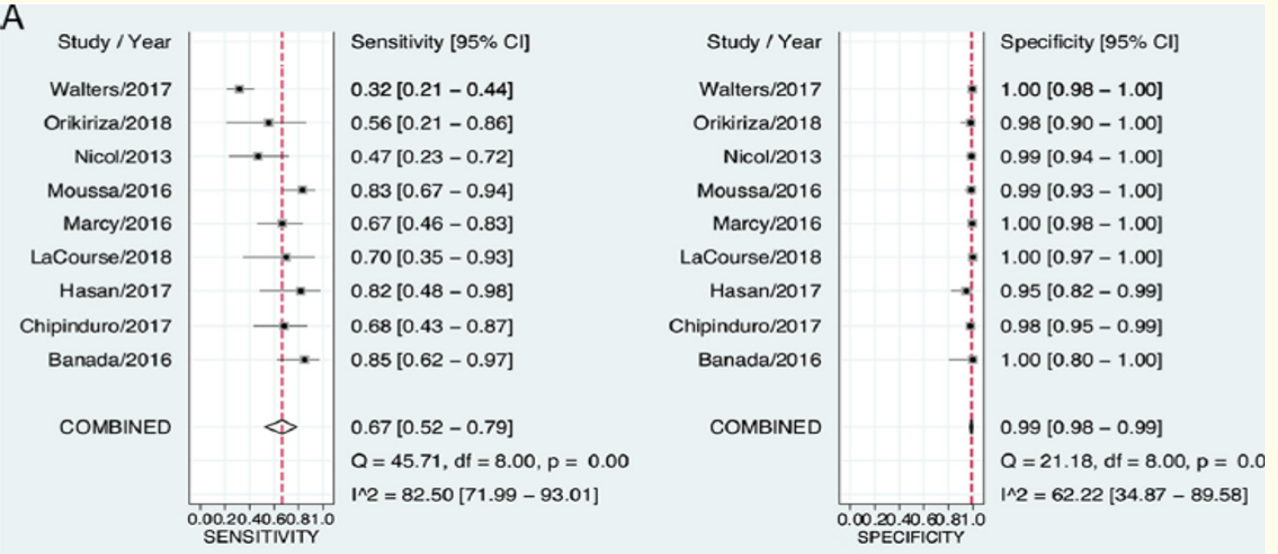

(A) Forest plots of stool Xpert's diagnostic performance compared to a microbiological reference standard of culture of Xpert positivity on respiratory samples.

B

B

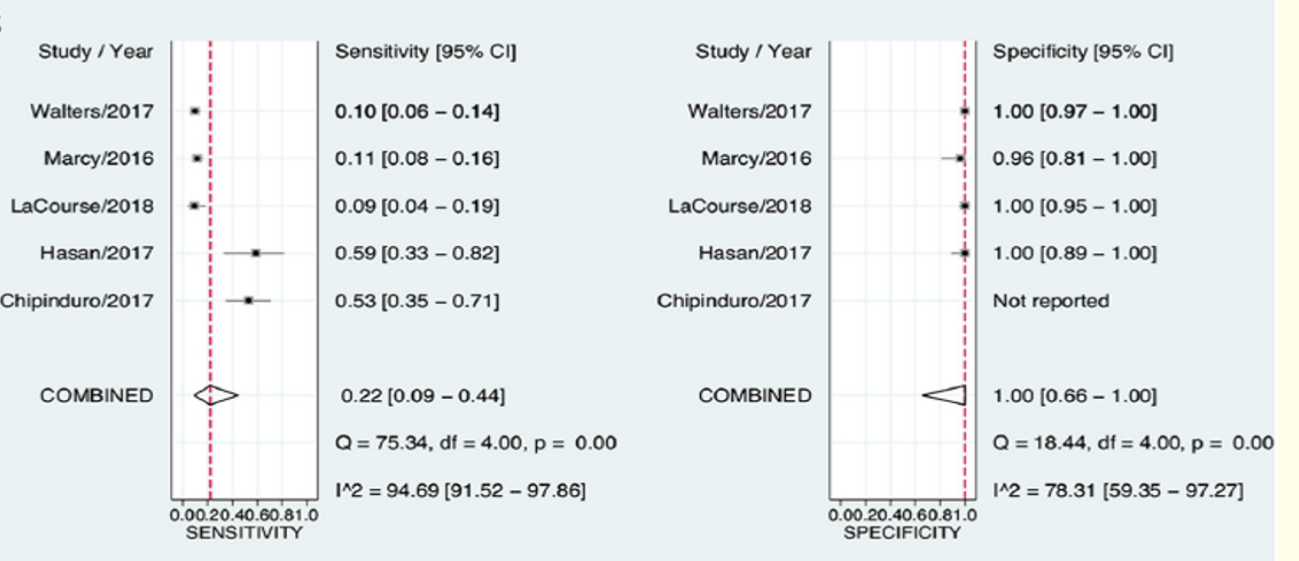

(B) Forest plots of stool xpert's diagnostic performance compared to a clinical reference standard of "likely / possible TB" or "unlikely TB".

C

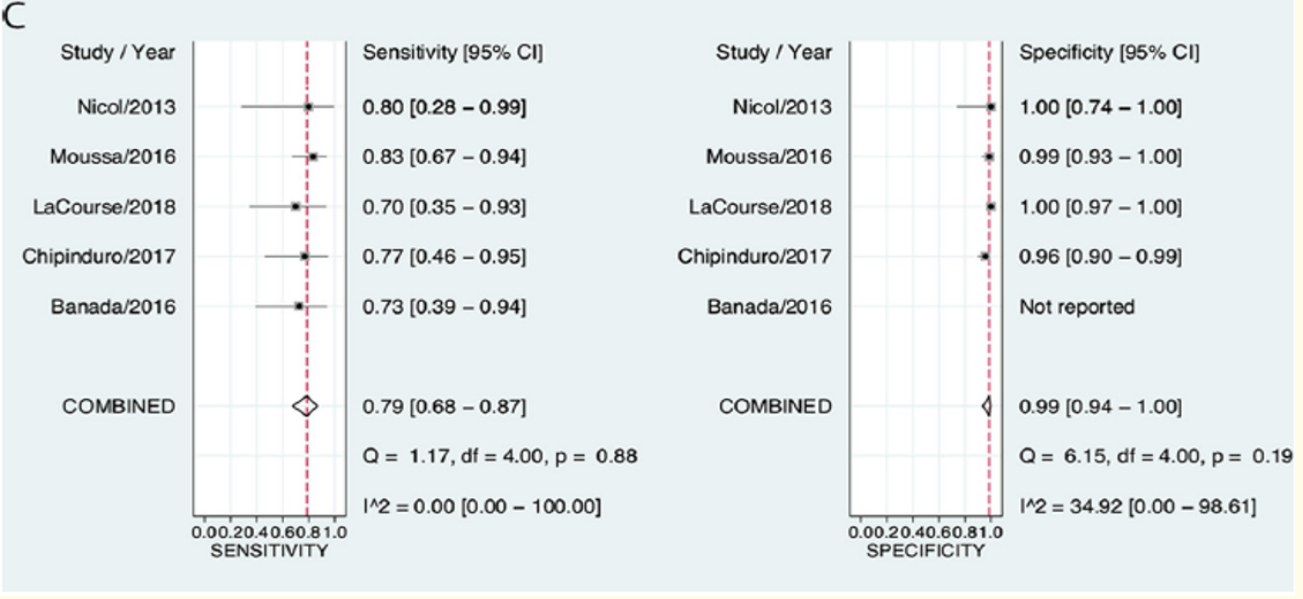

(C) Forest plots of diagnostic performance of stool xpert in children with HIV compared to a microbiological reference standard. 


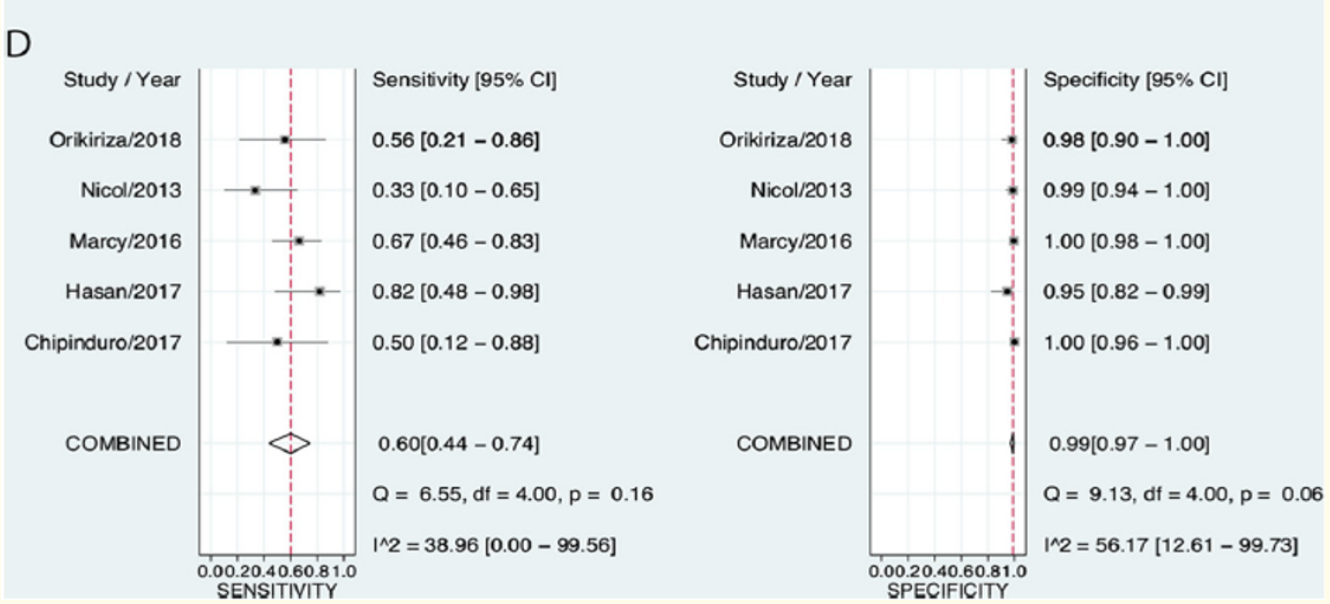

(D) Forest plots of diagnostic performance of stool xpert in HIV negative children compared to a microbiological reference standard.

\begin{tabular}{|l|c|c|c|c|c|c|}
\hline \multicolumn{1}{|c|}{ Comparison } & \multicolumn{3}{|c|}{ Main results } & \multicolumn{2}{c|}{$\begin{array}{c}\text { Results of sensitivity analysis excluding the study } \\
\text { that did not use culture as reference standard }\end{array}$} \\
\hline & $\begin{array}{c}\text { No. of studies } \\
\text { (no. of } \\
\text { children) }\end{array}$ & $\begin{array}{c}\text { Pooled } \\
\text { sensitivity } \\
\text { \% (95\% CI) }\end{array}$ & $\begin{array}{c}\text { Pooled } \\
\text { specificity \% } \\
(\mathbf{9 5 \%} \text { CI) }\end{array}$ & $\begin{array}{c}\text { No. of studies } \\
\text { (no. of children) }\end{array}$ & $\begin{array}{c}\text { Pooled } \\
\text { sensitivity } \\
\text { \%(95\% CI) }\end{array}$ & $\begin{array}{c}\text { Pooled } \\
\text { specificity } \\
\text { \%(95\% CI) }\end{array}$ \\
\hline $\begin{array}{l}\text { Stool xpert vs } \\
\text { microbiological } \\
\text { reference standard }\end{array}$ & $9(1681)$ & $67(52-79)$ & $99(98-99)$ & $8(1644)$ & $64(49-76)$ & $99(98-100)$ \\
\hline $\begin{array}{l}\text { Stool xpert vs clinical } \\
\text { reference standard }\end{array}$ & $5(869)$ & $22(92-98)$ & $100(66-100)$ & Not applicable & Not applicable & Not applicable \\
\hline $\begin{array}{l}\text { Stool xpert vs } \\
\text { microbiological reference } \\
\text { standard in HIV + children }\end{array}$ & $5(395)$ & $79(68-87)$ & $99(94-100)$ & $5(379)$ & $80(68-88)$ & $99(94-100)$ \\
\hline $\begin{array}{l}\text { Stool xpert vs microbiologi- } \\
\text { cal reference standard in } \\
\text { HIV -- children }\end{array}$ & $7(974)$ & $61(40-79)$ & $99(98-100)$ & Not applicable & Not applicable & Not applicable \\
\hline
\end{tabular}

\section{Table 1}

tion sequences), and the optimization of PCR and thermal-cycling parameters. The results are provided automatically in $77 \mathrm{~min}$ if the genetic material is amplified or in $66 \mathrm{~min}$ if it is not. The system classifies MTUBC detection in the following semiquantitative results: high, medium, low, very low, and a new category named trace, and it classifies RIF resistance as detected, not detected, or indeterminate [9].

\begin{tabular}{|c|c|c|c|c|c|c|c|c|c|c|c|c|}
\hline \multirow[t]{2}{*}{ Author } & \multirow{2}{*}{ Site } & \multirow[t]{2}{*}{ Year } & \multirow[t]{2}{*}{ Type } & \multirow[t]{2}{*}{ No./Age } & \multirow{2}{*}{$\begin{array}{l}\text { Hiv } \\
\%\end{array}$} & \multirow{2}{*}{ Prevalence\% } & \multirow[t]{2}{*}{ Sample } & \multirow{2}{*}{ Reference } & \multicolumn{2}{|c|}{$\begin{array}{c}\text { Sensitivity } \\
\text { Sn \% }\end{array}$} & \multicolumn{2}{|c|}{$\begin{array}{c}\text { Specificity } \\
\text { Sp \% }\end{array}$} \\
\hline & & & & & & & & & Ultra & Xpert & Ultra & Xpert \\
\hline Nicol & $\begin{array}{l}\text { South } \\
\text { Africa }\end{array}$ & $2012-17$ & $\mathrm{R}$ & $367<15$ & 19 & $23(83 / 367)$ & $\begin{array}{l}\text { Frozen } \\
\text { Sputum }\end{array}$ & $\begin{array}{c}\text { Culture, } \\
\text { GEU }\end{array}$ & 74 & 63 & 97 & 100 \\
\hline Sabi & Tanzania & $2011-12$ & $\mathrm{R}$ & $2150.5-16$ & 52 & $13(28 / 215)$ & $\begin{array}{l}\text { Frozen } \\
\text { Sputum }\end{array}$ & Culture & 67 & NR & 98 & NR \\
\hline
\end{tabular}

Table 2 
Stool is a promising sample matrix for pediatric TB tests, because MTB may be swallowed and passed into a child's stool where it can be easily sampled. MTB is difficult to routinely culture from stool, because other rapidly growing bacteria may overgrow the slowly replicating $M$. tuberculosis bacillus. Nucleic acid based amplification tests (NAAT) can detect MTB in stool with good sensitivity. The Xpert MTB/RIF (Xpert) assa, recommended to detect TB in sputum, has also been exploited for extra-pulmonary samples. In previous TB detection studies performed on stool, the Xpert assay detected MTB with a sensitivity of $25-69 \%$ and a specificity of $91.7-100 \%$. However, the stool processing protocols required centrifugation, which would not be suitable for point of care testing in resource-limited environments. Xpert stool tests also generated unacceptably large numbers of errors (13 - 25\%), probably from sample clogging the Xpert assay cartridge or due to PCR inhibition from stool components. The poor sensitivity of $<50 \%$ reported in some Xpert stool studies could have been due to retained PCR inhibitors, but may also have been due to the low amounts of sample that were tested $(<0.2 \mathrm{~g})$. Assay sensitivity could be increased if methods were available for testing larger quantities of stool without introducing PCR inhibitors [10-20].

A simple method for processing stool samples for Xpert MTB/ RIF testing using minimal equipment has been announced at the 49th Union World Conference on Lung Health and could enable the identification of thousands of children with tuberculosis (TB) and multidrug-resistant TB (MDR-TB) disease - drastically reducing the numbers of children under five dying from TB [19].

This groundbreaking work shows that a simple and low-cost method, using similar processing steps as are applied for sputum, can diagnose TB at any GeneXpert site using stool samples, and can even diagnose additional children. The method has the potential to make a bacteriological diagnosis of TB in children at the lowest levels of health care $[1,20]$.

In the early 1990s, when RNTCP recommended two AFB smear examinations to be equal to culture for case finding and since majority of AFB smear preparation was carried out on open laboratory benches without biosafety hoods, we developed a sputum processing method which rendered $\mathrm{M}$ tb innocuous and floated them on a layer of xylene. We intend to adopt this method for simple stool processing wherein $0.6 \mathrm{G}$ of stool would be homogenized in $10 \mathrm{ml}$ of DW in a screw capped flat bottom tube and then $1 \mathrm{ml}$ of xylene will be mixed. The tube will be left undisturbed for $30 \mathrm{~min}-$ utes to allow debris to settle and xylene to form a layer on top of the DW. Xylene being a lipid solvent will extract out the lipid rich M tb (if present) in the sample. This $1 \mathrm{ml}$ will be mixed with $1 \mathrm{ml}$ of
Sample buffer of Gene Xpert and two ml of material will be added to a cartridge of Gene Xpert Ultra [21].

In laboratory studies using Gene Xpert it has been documented that the level of detection for Xpert on Macaque stools was determined to be approximately 1000 colony forming units (CFU) / ml, whereas in respiratory samples it is approximately $100 \mathrm{CFU} / \mathrm{ml}$. Given this higher detection threshold of stool Xpert when using Gene Xpert MTB/RIF on stool compared to respiratory samples, stool Xpert would only detect a proportion of children with bacteriologically confirmed TB. However, with better sample preparation and use of Gene Xpert Ultra cartiage (which is atlteast 8 times more sensitive than GE MTB/RIF) the results now could be far different [4].

Furthermore, when direct comparison was made with diagnostic potential of a single Gastric Aspirate or IS sample, stool was not substantially inferior. Stool Xpert had a substantial incremental yield over culture of the first respiratory sample, indicating that although stool Xpert will miss some cases, the total number detected would be comparable to a single respiratory sample, which is more invasive than stool testing [4].

Stool collection is potentially a more feasible and acceptable alternative to collecting respiratory samples, and can occur at home. Mycobacterium tuberculosis (M.tb) in swallowed sputum is recoverable from stool samples by culture or molecular methods. Stool does not require sophisticated collection equipment and poses low TB infection risk to healthcare workers. In particular, stool is easy to obtain from young children, a high-risk group for severe TB and where diagnosing TB is the most challenging. In critically ill children, stool collection may be a safer alternative to invasive sputum collection procedures [22].

\section{Conclusions}

Stool Xpert can rapidly confirm TB in children who present with radiologic findings suggestive of severe TB. In resource-limited settings where children frequently present with advanced disease, Xpert on stool samples could improve access to rapid microbiological confirmation and initiation of appropriate treatment in developing countries like India [22,23].

\section{Bibliography}

1. Banada PP., et al. "A novel sample processing method for rapid detection of tuberculosis in the stool of pediatric patients using the xpert MTB/RIF assay". PLOS one 11.3 (2016): 0151980. 
2. Kitai I., et al. "Diagnosis and management of pediatric tuberculosis in Canada". CMAJ (2017): E11.

3. Loos V., et al. "Alternative sputum collection methods for diagnosis of childhood intrathoracic tuberculosis: a systematic literature review". Archives of Disease in Childhood 104.7 (2019): 629-635.

4. Walters E., et al. "Xpert MTB/RIF on stool is useful for the rapid diagnosis of tuberculosis in young children with severe pulmonary disease". The Pediatric Infectious Disease Journal 36.9 (2017): 837-843.

5. Cepheid press release (2019).

6. Graham SM., et al. "Clinical case definitions for classification of intrathoracic tuberculosis in children: an update". Clinical Infectious Diseases 61(2015): S179.

7. Brown M., et al. "Prospective study of sputum induction, gastric washing, and bronchoalveolar lavage for the diagnosis of pulmonary tuberculosis in patients who are unable to expectorate". Clinical Infectious Diseases 44.11 (2007): 1415-1420.

8. MacLean E., et al. "Diagnostic accuracy of stool Xpert MTB/RIF for detection of pulmonary tuberculosis in hildren: a systematic review and metaanalysis". Journal of Clinical Microbiology 57.6 (2019): e02057.

9. Perez-Risco D., et al. "Evaluation of the xpert MTB/RIF ultra assay for direct detection of Mycobacterium tuberculosis complex in smear negative extrapulmonary samples". Journal of Clinical Microbiology 56.9 (2018): e00659.

10. Khechine AE., et al. "Detection of Mycobacterium tuberculosis complex organisms in the stools of patients with pulmonary tuberculosis". Microbiology 155 (2009): 2384-2389.

11. Nicol MP., et al. "Xpert MTB/RIF testing of stool samples for the diagnosis of pulmonary tuberculosis in children". Clinical Infectious Diseases 57.3 (2013): e18.

12. Welday SH., et al. "Stool as appropriate sample for the diagnosis of Mycobacterium tuberculosis by Gene Xpert test". Open Journal of Respiratory Diseases 4 (2014): 83.

13. Moussa HS., et al. "Gene xpert for direct detection of Mycobacterium tuberculosis in stool specimens from children with presumptive pulmonary tuberculosis". Annals Clin Lab Sciences 46.2 (2016): 198-203.
14. Chipinduro M., et al. "Stool xpert MTB/RIF test for the diagnosis of childhood pulmonary tuberculosis at primary clinics in Zimbabwe". International Journal of Tuberculosis and Lung Disease 21.2 (2017): 161-166.

15. Memon SS., et al. "Diagnostic accuracy of xpert MTB/RIF assay in stool samples in intrathoracic childhood tuberculosis". Journal of Tuberculosis and Therapeutics 3 (2018): 2.

16. Walters E., et al. "Molecular detection of Mycobacterium tuberculosis from stools in young children by use of a novel centrifugation free processing method". Journal of Clinical Microbiology 56 (2018): e00781.

17. Walters E., et al. "Stool culture for diagnosis of pulmonary tuberculosis in children". Journal of Clinical Microbiology 55.12 (2017): 3355-3365.

18. Medecins sans frontiers. Stool gene xpert MTB/RIF assay standard operating procedure.

19. The 39th Union World Conference on Lung Health. The Hague, Press release (2018): 24-27.

20. Drancourt M. "Please no more gastric aspirate to diagnose pulmonary tuberculosis in children". Clinical Infectious Diseases 65 (2017): 2158.

21. Rattan A., et al. "Evaluation of a safe sputum processing method for detecting tuberculosis". Journal of Clinical Pathology 47.5 (1994): 411.

22. Atherton RR., et al. "Xpert MTB/RIF ultra for tuberculosis testing in children: a mini review and commentary". Frontiers in Pediatrics 7 (2019): 34.

23. Mesman AW., et al. "Detection of Mycobacterium tuberculosis in pediatric stool samples using TruTip technology". BMC Infectious Diseases 19 (2019): 56.

Volume 2 Issue 10 October 2019

(C) All rights are reserved by Ashok Rattan. 